\title{
Florence Bergeaud-Blackler (éd.), Les sens du Halal. Une norme dans un marché mondial
}

CNRS Éditions, coll. « Alpha », Paris, 2015.

\section{Katerina Seraïdari}

\section{(2) OpenEdition}

\section{Journals}

Édition électronique

URL : http://journals.openedition.org/assr/28184

DOI : $10.4000 /$ assr.28184

ISSN : $1777-5825$

Éditeur

Éditions de l'EHESS

Édition imprimée

Date de publication : 31 décembre 2016

Pagination : 267

ISSN : 0335-5985

Référence électronique

Katerina Seraïdari, « Florence Bergeaud-Blackler (éd.), Les sens du Halal. Une norme dans un marché mondial », Archives de sciences sociales des religions [En ligne], 176 | octobre-décembre 2016, mis en ligne le 17 juillet 2017, consulté le 24 septembre 2020. URL : http://journals.openedition.org/assr/ 28184 ; DOI : https://doi.org/10.4000/assr.28184

Ce document a été généré automatiquement le 24 septembre 2020.

(c) Archives de sciences sociales des religions 


\title{
Florence Bergeaud-Blackler (éd.), Les sens du Halal. Une norme dans un marché mondial
}

CNRS Éditions, coll. « Alpha », Paris, 2015.

\author{
Katerina Seraïdari
}

\section{RÉFÉRENCE}

Florence Bergeaud-Blackler (éd.), Les sens du Halal.

1 Ce volume, qui couvre un grand nombre des questions autour de l'espace alimentaire musulman, montre comment le halal, par une extension de son sens, est devenu un «mode de vie» et une éthique de l'action. La première partie du livre (et la plus importante en nombre de contributions, la deuxième partie n'étant constituée que de trois articles) examine, en effet, comment le halal s'est progressivement transformé en «espace normatif». Né dans les contextes diasporiques de l'islam et mobilisé de manière de plus en plus significative depuis les années 1990, le halal a une histoire, comme nous le rappellent les quatre premiers articles qui adoptent une approche plutôt historique. Burak Onaran analyse comment la colonisation et l'occidentalisation ont contribué à la propagation de la consommation de viande, qui est alors considérée " comme un aliment essentiel pour l'activité humaine, pour une armée plus puissante et pour une nation plus productive et musclée » (p. 41). Toutefois, la différence dans les méthodes d'abattage conduit les familles musulmanes bordelaises dans les années 1990 à distinguer la viande des « chrétiens », plus rouge, d'une viande halal à la teinte rosée, comme l'éditrice du volume l'explique dans le premier de ses deux articles (p.93). Devenant un « espace de licéité » à construire et à étendre jusqu'aux cosmétiques, aux crédits financiers et aux paquets vacances, aux sex-shops et à la médecine, le halal n'est plus seulement une affaire de viande: l'opposition entre ce qui est halal ou harām 
(interdit) dépasse les frontières religieuses et alimentaires pour entrer dans le domaine de l'industrie des loisirs et dans l'organisation du monde économique.

2 Si le mot fait son entrée dans les dictionnaires français dès 1987 (p. 288), le livre permet de découvrir les paramètres qui influent sur ce travail sémantique: l'engouement récent pour les produits bio et le mouvement écologique véhiculant des représentations et des discours réappropriés par certains courants islamiques, en ce qui concerne par exemple l'envie de consommer une «viande heureuse » (des moutons sacrifiés pour la fête de l'Aïd ayant parfois reçu un traitement homéopathique la veille et d'aromathérapie le jour même, comme Jean-Paul Ferrié le précise dans sa contribution) ; les évolutions dans le domaine de l'alimentation, avec le quality turn des années 1990 (p.125) et le souci de la qualité des aliments et de leur traçabilité (« de la fourche à la fourchette ») qui conduit à une nouvelle "éthique de consommation »; mais aussi les références au marché cacher qui fonctionne comme un modèle et comme un point de comparaison pour le développement du marché halal. D'ailleurs, l'idée de Manuel Valls, après les attentats de janvier 2015, d'un financement du culte musulman par la viande halal, a été calquée sur le modèle juif de la cacherout, comme le montre Florence Bergeaud-Blackler dans un article de Libération (3 mars 2015).

3 Le fait que le halal tende à être transformé en "label de qualité » ne montre pas seulement dans quelle mesure il est traversé par le langage du marketing, mais aussi comment le marché mondial utilise les appartenances religieuses et identitaires pour accroître l'offre et élargir la demande. Le halal est un "conte néo-libéral du $\mathrm{xxI}^{\mathrm{e}}$ siècle ", comme le suggère l'éditrice du volume dans le titre de son second article. Les pays du sud-est asiatique, avec en tête la Malaisie, ont joué un rôle considérable dans cette expansion du marché à partir des années 2000 : le principal promoteur de la sensibilisation au halal reste la multinationale Nestlé, implantée depuis 1912 en Malaisie qui sert, dans le contexte actuel, de pays test (p.179). Ce qui complique les choses est le fait que, si l'État français est porté garant des directives bio, il ne peut pas jouer ce même rôle en ce qui concerne les directives halal, puisque cette référence qui semble aller de soi, c'est-à-dire la «loi islamique ", n'a pas d'existence en droit international ou commercial. La contribution de Stéphane Papi insiste sur le fait que les lois religieuses sont sans valeur juridique (p. 191).

4 Le volume trace donc, dans un premier temps, l'histoire de l'halalisation et ses racines théologiques, avant de se tourner, dans un deuxième temps, vers l'étude du halal en tant que phénomène lié à l'industrialisation et à la globalisation. Les deux articles de l'éditrice parviennent à baliser cette évolution, qui resterait beaucoup plus floue s'il n'y avait que les textes des différents contributeurs. En effet, les différents cas de figure, qui vont de l'esturgeon iranien au Green Halal, des produits financiers islamiques à la greffe d'organes, et de l'abattage rituel au halal carcéral (ce qui pose la question de l'offre institutionnelle, non seulement dans les prisons, mais aussi dans les écoles et l'armée, et qui met en lumière les interactions permanentes entre les institutions et l'économie de marché) révèlent surtout l'éventail des pratiques et des discours autour de ce sujet, sans proposer de lignes explicatives claires. Les articles de l'éditrice remédient, de manière importante, à ce problème. De ce point de vue, l'ouvrage offre plus une vue sur la profusion des sens du halal qu'une analyse de sa place dans le marché mondial - d'ailleurs, plusieurs articles ne traitent pas du tout de cela : outre le texte sur le halal carcéral, c'est aussi le cas de certains articles dont l'approche est historique, et de ceux qui s'intéressent à la sexualité des musulmans. L'ouvrage paraît 
ainsi, plusieurs fois, décentré : comme si pour illustrer la confusion qui règne autour du halal, il fallait la laisser entrer dans le recueil, par un assemblage de case studies. En effet, dans l'introduction, l'éditrice explique que le but du colloque qui a conduit à cette publication a été de "faire émerger la plurivocité des sens" du halal. Elle distingue néanmoins trois grands usages: les usages savants et théologiques, les usages marchands qui posent la question de la qualité, et les usages diasporiques qui font du halal un espace normatif (p. 10-11). Les deux premiers usages, qui renvoient à une multitude d'acteurs dont les actions sont en constante interaction, suggèrent autant des différentiations que des imbrications : législateur divin qui donne sens à la notion d'obligation morale versus législateurs actuels essayant de réguler le marché mondial ; définitions religieuses du halal versus ses définitions industrielles. Quant aux usages diasporiques, ils concernent autant le pratiquant que le consommateur qui change ses habitudes de consommation selon l'offre et les modes qu'elle introduit. Dans ce cadre, des acteurs importants, comme l'État, interviennent tant dans les usages marchands que dans les usages diasporiques.

5 L'ouvrage propose donc une « réflexion plurielle » et interdisciplinaire, avec toute la richesse et, dans un moindre degré, les défauts que cela implique. Il nous fait ainsi passer d'une figure typiquement italienne, celle de l'imam boucher, que Khalid Rhazzali analyse (p. 212), aux effets néfastes du halal sur le petit commerce en Turquie, étant donné que celui-ci favorise un mode de vente rationalisé et, par conséquent, le développement des supermarchés, comme Gérard Groc le suggère (p. 243). Il donne beaucoup d'éléments sur l'abattage industriel, tout en montrant la tendance actuelle de préférer, parmi les musulmans qui promeuvent un «éco-islam », l'idée de sacrifice à celle d'abattage, selon Manon Istasse (p. 128). De même, il ouvre de nombreuses pistes de réflexion, par exemple, sur la relation entre le halal et le "terroir » : associé à la particularité productive de chaque région, ce dernier constitue une autre voie dans le développement du marché mondial alimentaire. La question qui se pose est de savoir si le halal fait figure de contretype par rapport au «terroir ». Alors que le discours du Front National que Chantal Crenn et Pascal Tozzi analysent (p. 300, note 15) va dans ce sens, des éléments concernant le développement d'un «éco-islam » montrent plutôt des convergences. Des comparaisons de ce genre permettent de réfléchir non seulement sur les mécanismes d'éthicisation du halal, mais surtout sur les mécanismes d' éthicisation d'autres domaines marchands, beaucoup moins liés à la religion, comme le terroir, et plus généralement, sur la manière dont le marché mondial génère de nouveaux espaces normatifs et éthiques. 